See discussions, stats, and author profiles for this publication at: https://www.researchgate.net/publication/306928586

\title{
Cardiorespiratory fitness effect may be under-estimated in 'fat but fit' hypothesis studies
}

Article in Annals of Human Biology · April 2017

DOI: 10.1080/03014460.2016.1229029

CITATIONS

9

9 authors, including:

Danilo R P Silva

Universidade Federal de Sergipe

63 PUBLICATIONS 464 CITATIONS

SEE PROFILE

Paul Collings

University of Cambridge

45 PUBlicationS 454 CITATIONS

SEE PROFILE

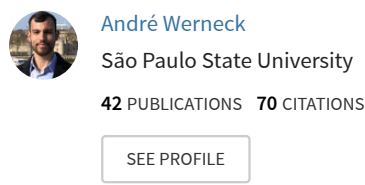

(6) David Ohara

State University of Santa Cruz

19 PUBLICATIONS 131 CITATIONS

SEE PROFILE

Some of the authors of this publication are also working on these related projects:

Post-Doctoral Fellowship, Columbia University, New York Obesity Research Centre, St. Luke's Roosevelt Hospital and Technical University of Lisbon, View project

COMPETITIVE EFFECT BETWEEN FAT FREE MASS AND ADIPOUS TISSUE ON BONE MINERAL DENSITY GAINS OF INFERIOR MEMBERS OF SPORTS ADOLESCENTS: ABCD-

Growth Study View project 


\title{
Cardiorespiratory fitness effect may be under-estimated in 'fat but fit' hypothesis studies
}

\author{
Danilo R. Silva ${ }^{a}$, André O. Werneck ${ }^{a}$, Paul J. Collings ${ }^{b}$, David Ohara ${ }^{a}$, Rômulo A. Fernandes ${ }^{c}$, Décio S. Barbosa ${ }^{d}$, \\ Enio R. V. Ronque ${ }^{a}$, Luís B. Sardinha ${ }^{e}$ and Edilson S. Cyrino ${ }^{a}$

\begin{abstract}
${ }^{\text {a} S t u d y ~ a n d ~ R e s e a r c h ~ G r o u p ~ i n ~ M e t a b o l i s m, ~ N u t r i t i o n, ~ a n d ~ E x e r c i s e ~-~ G E P E M E N E, ~ S t a t e ~ U n i v e r s i t y ~ o f ~ L o n d r i n a ~-~ U E L, ~ L o n d r i n a, ~ B r a z i l ; ~}$ ${ }^{b}$ Bradford Institute for Health Research, Bradford NHS Foundation Trust, Bradford, UK; 'Scientific Research Group Related to Physical Activity (GICRAF), Laboratory of Investigation in Exercise (LIVE), Department of Physical Education, São Paulo State University (UNESP), Presidente Prudente, Brazil; ${ }^{d}$ Department of Pathology, Clinical and Toxicological Analysis, Center of Health Sciences, University Hospital, State University of Londrina - UEL, Londrina, Brazil; ${ }^{2}$ Exercise and Health Laboratory, Faculty of Human Movement, University of Lisbon, Portugal
\end{abstract}

\begin{abstract}
Background: Both cardiorespiratory fitness and body fat have been independently related to metabolic syndrome in adolescents; however, the strength of these relationships seems to be dependent on the outcome composition.

Aim: To analyse the relationship between cardiorespiratory fitness and body fat combined with different indicators of metabolic risk in adolescents.

Subjects and methods: The sample was composed of 957 adolescents ( $58.7 \%$ girls). Cardiorespiratory fitness was obtained using the 20-metre shuttle run test and skinfold thickness was collected for body fat estimation. Metabolic risk score was calculated from waist circumference, systolic and diastolic blood pressure, glucose, HDL cholesterol and triglycerides measurements and an alternative outcome without the central obesity indicator was adopted. Chronological age and somatic maturity were used as covariates.

Results: Higher metabolic risk was observed in the highest fat/lowest fit adolescents $(p<.05)$, regardless of sex and outcome. In the regression models, for full metabolic risk score, body fat presented higher coefficients compared to cardiorespiratory fitness in both sexes (boys: 0.501 vs - 0.097; girls: 0.485 vs -0.087); however, in the metabolic risk without waist circumference, the coefficients became closer (boys: 0.290 vs -0.146 ; girls: 0.265 vs -0.120 ), with a concomitant decrease in body fat and increase in cardiorespiratory fitness coefficients.

Conclusion: These findings suggest that body fat is strongly related to cardiovascular risk, but, when the outcome is calculated without the central obesity indicator, cardiorespiratory fitness becomes more related to metabolic risk.
\end{abstract}

\section{ARTICLE HISTORY}

Received 20 October 2015

Accepted 27 July 2016

Published online 22 Septem-

ber 2016

\section{KEYWORDS}

Metabolic syndrome; obesity; $\mathrm{VO}_{2 \text { max }}$ f fatness

\section{Introduction}

Nowadays, obesity is one of the biggest challenges to public health. The deposit of body fat is related to cardiovascular risk, predominantly in adults; however, the pathological condition of obesity introduced in early ages has been identified as a precursor of several chronic diseases (Lee, 2009). In addition, studies performed over the past few decades have indicated that cardiorespiratory fitness could be an important protective factor for metabolic risk (Machado-Rodrigues et al., 2014; Stabelini Neto et al., 2011), including attenuating the negative effect of obesity (Eisenmann et al., 2007), a paradigm denominated 'fat but fit' (Barlow et al., 1995).

Broadly speaking, the main results of this theory indicate the importance of cardiorespiratory fitness, although the majority of the studies highlight body fat as the most relevant factor for predicting negative outcomes (DuBose et al., 2007; Eisenmann et al., 2005, 2007; Kwon et al., 2010). Methodologically, the most commonly used indicator of cardiovascular risk is composed of metabolic syndrome variables together with an indicator of abdominal obesity. Eisenmann (2007), for example, in a review paper observed that three of four 'fat but fit' studies with children and adolescents included waist circumference in their metabolic risk score.

However, given the more direct relationship between body fat and abdominal obesity, the independent relationship between cardiorespiratory fitness and metabolic risk is not clear, nor is its protective power over outcomes with a greater latency period, but similarly important for cardiovascular risk, such as high fasting glucose and dyslipidemia (Eisenmann et al., 2004). In addition, the lack of control over somatic maturity, which is an important variable in the phase of life and is related to both fatness and fitness, can confound the previous results.

Thus, in order to discuss these issues and target long-term interventions, the aim of this study was to analyse the relationship of cardiorespiratory fitness and body fat with metabolic risk, both with and without a central obesity indicator in adolescents. 


\section{Methods}

\section{Sample}

This was a school-based epidemiological study, conducted in adolescents aged between 10-16 years, matriculated in public schools from Londrina/PR/Brazil in 2011. Londrina has 506,701 inhabitants, a human development index (HDI) of 0.778 , and a gross domestic product (GDP) per capita of US\$8,530.77 (BIGS, 2013).

The sampling process was conducted in two stages. First, all the public schools of the city were separated into regions (north, south, east, west, and centre). Next, two schools were randomly selected from each region. Finally, classes (morning and evening) were randomly selected from the previously chosen schools and within these classes all students were invited to participate in the study. Students who were taking prescription medicine, were undergoing treatment for any disease or did not return the consent form signed by parents were excluded from the sample. All study procedures were approved by the local Ethics Committee, according to the Declaration of Helsinki.

\section{Body fat}

Body fat measurements were obtained through subscapular and tricipital skinfold thicknesses, which were measured by a trained evaluator using a Lange ${ }^{\circledR}$ caliper, properly calibrated (precision $=0.5 \mathrm{~mm}$ ), according to the recommendations of Harrison et al. (1988). For quality control, a pilot project was conducted with 90 adolescents who did not compose the sample of the present study; however, their characteristics were similar with regard to somatic maturation and body fat. The technical errors of measurement (TEM) were $4.8 \%$ and $3.5 \%$ for subscapular and tricipital skinfolds, respectively. From this information, the percentage of body fat was calculated according to the equations proposed by Boileau et al. (1985). To determine the cut-points, after distribution of the sample into quartiles, the adolescents who presented a body fat percentage in the highest quartile (boys $>23.3 \%$, girls $>30.2 \%$ ) were considered as fat.

\section{Cardiorespiratory fitness}

The 20-metre shuttle run test (Leger \& Lambert, 1982), performed on sports courts, was used to estimate cardiorespiratory fitness. The peak oxygen consumption, in $\mathrm{ml} / \mathrm{kg} / \mathrm{min}$, was estimated according to the equations proposed by Leger et al. (1988). Similar to body fat, we divided the sample into quartiles and the adolescents in the lowest quartile of $\mathrm{VO}_{2}$ peak (Boys $<39.0 \mathrm{ml} / \mathrm{kg} / \mathrm{min}$, girls $<36.0 \mathrm{ml} / \mathrm{kg} / \mathrm{min}$ ) were considered as unfit.

\section{Metabolic risk score}

The following variables: waist circumference; systolic and diastolic blood pressure; glucose; HDL cholesterol and triglycerides, were adopted to provide the metabolic risk score.
Waist circumference was measured according to the recommendations of Katzmarzyk et al. (2004), using an anthropometric tape with a precision of $0.1 \mathrm{~cm}$. Blood pressure measurements were performed on the right arm, after a rest period of 10 minutes, using an automatic OMRON/ HEM-742, previously validated for adolescents (Christofaro et al., 2009). Two measurements were taken, with a 2minute interval; if the difference between the measurements was greater than $10 \mathrm{mmHg}$ for diastolic blood pressure (DBP) or systolic blood pressure (SBP), a third measurement was performed. In this case, the two closest measurements were considered and the final blood pressure score was determined as the arithmetic mean of these two values.

The laboratory analyses were carried out at the Laboratory of Clinical Chemistry, University Hospital, State University of Londrina. The blood samples were collected, after fasting for 12 hours, in a serum tube (without anticoagulant) and a tube containing anticoagulant and fluoride, as a preservative, for the determination of glucose. To analyse HDL and triglycerides, blood was collected from the antecubital vein while participants remained seated. After the collection procedure, the tubes with samples were centrifuged at $3000 \mathrm{rpm}$ for 5 minutes at $4{ }^{\circ} \mathrm{C}$ to separate the plasma and serum. The analyses were realised immediately after the separation through a biochemical auto-analyser system, Dimension RxL Max - Siemens Dade-Behring. Intra-assay CV were below 10\% for all laboratory analysis.

To obtain the aggregate metabolic risk score, we calculated the arithmetic mean of the individual risk factors of metabolic syndrome $z$-score values (HDL [reverse signal], triglycerides, fasting glucose, systolic blood pressure and diastolic blood pressure). In addition, for analysis purposes, we created a score without waist circumference (the factor most directly related to body fat indicators).

\section{Somatic maturation}

Peak height velocity was calculated using the equations proposed by Mirwald et al. (2002) from body weight, height and trunk-cephalic height measurements. The age of peak height velocity (APHV) was obtained by subtracting the peak height velocity from the chronological age and used as the somatic maturity indicator.

\section{Statistical analyses}

For characterising the sample, data were presented as mean and standard deviation. Homogeneity of variances was tested using the Levene's test. For the comparisons of different variables between the groups, ANCOVA (adjusted by sex, chronological age and APHV) was used with the Bonferroni post-hoc test. The Chi-square test was used for the distribution of sexes into the categories and, finally, linear regression was used for verifying the relationship (crude and adjusted by chronological age and APHV) between independent variables and risk score. All the analyses were processed using SPSS 17.0 software, with a significance of $p<.05$. 
Table 1. Profile of adolescents according to adiposity and cardiorespiratory fitness categories.

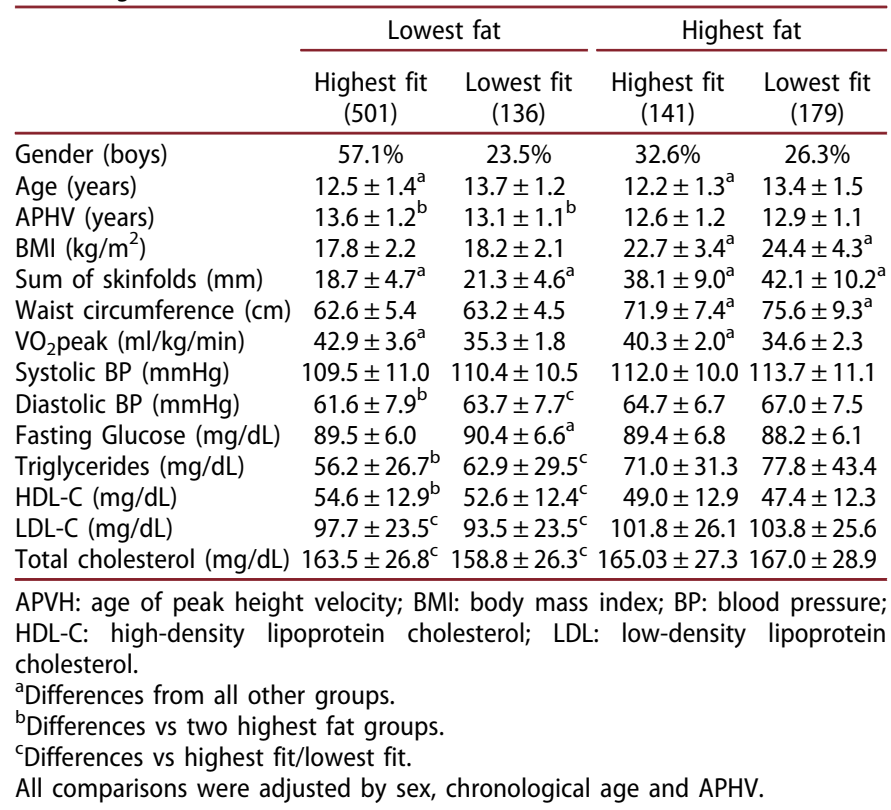

\section{Results}

Of the 1,395 adolescents initially evaluated, 379 were outside the age range for the study (10-16 years) and 59 did not complete the evaluations used in this study and were excluded. Thus, the final sample was composed of 957 adolescents; $58.7 \%$ girls, $66.3 \%$ of low socio-economic status and $22 \%$ overweight.

Table 1 presents the characteristics of the sample and comparisons between the four groups. In general, the highest fat/lowest fit group presented the highest average values of risk factors compared with the other groups. Aside from the variables inherent to the categorisation of the groups, we observed differences between the intermediate groups (lowest fat/lowest fit and highest fat/highest fit) only for glucose, for which the group with lowest fat/lowest fit presented higher averages compared to the highest fat/highest fit group.

The metabolic risk scores, with and without waist circumference, of the four groups, are presented in Figure 1. In the general score, independent of sex, the highest fat/lowest fit group presented higher risk values when compared to the other groups and the lowest values were observed in the lowest fat/highest fit group. When the waist circumference variable was removed, the sexes demonstrated differences. In girls, the lowest values were observed in the lowest fat/highest fit group, when compared with the other groups. On the other hand, in boys, the differences were only observed between the extreme groups; there were no differences between any intermediate groups.

Table 2 presents the linear regression models for the analysis of the independent relationship between exposures and metabolic risk (with and without waist circumference). After adjusting for chronological age and APHV, we observed that the total risk score presented a higher coefficient of determination when compared to the score without waist circumference; however, both variables related independently with the outcome, in boys and girls, independent of a central obesity indicator.

\section{Discussion}

The main results of this study suggest that body fat and cardiorespiratory fitness are independently related to cardiovascular risk, even when waist circumference is removed from the risk score. It was observed that body fat was the leading predictor of risk, but its effect was attenuated by the insertion of cardiorespiratory fitness into the analysis. Moreover, in the risk score calculated without the central indicator of adiposity, cardiorespiratory fitness demonstrated proportionally higher coefficients than the initial model, when compared to body fat, whereas no difference between intermediate groups (highest fat/highest fit and lowest fat/ lowest fit) was observed. These findings have important implications for understanding the development of pathological outcomes in young people and developing interventions for the treatment and prevention of chronic diseases.

In addition to gathering evidence on the negative effects of high body fat and low cardiorespiratory fitness on health (Farrell et al., 2007), studies conducted in recent decades have tried to understand the interaction between these variables in the prediction of cardiovascular risk (Barlow et al., 1995), using cross-tabulated groups and multivariate models. The key mechanisms that justify this approach relate to higher oxidative capacity and synthesis of mitochondrial proteins in the highest fit individuals, which provide protection from the negative outcomes of elevated body fat (Wisloff et al., 2005).

Corroborating previous studies, we observed a gradual relationship between the fat/fit categories and cardiovascular risk, highlighted in the differences between the extreme categories (highest fat/lowest fit vs lowest fat/highest fit). These findings support the 'fat-but-fit' hypothesis (Barlow et al., 1995) in which cardiorespiratory fitness could attenuate part of the risk arising from high body fat (Eisenmann, 2007), which has been discussed in different populations (Barlow et al., 1995; DuBose et al., 2007; Eisenmann et al., 2005; Kwon et al., 2010). Early studies found similar results adopting different indicators of adiposity (Andersen et al., 2008; Cummings et al., 2010; Eisenmann et al., 2007; Kuk \& Lee, 2010; Silva et al., 2013; Suriano et al., 2010), however, few of these controlled for biological maturation, which seems to significantly influence body fat as well as cardiorespiratory fitness (Malina et al., 2004).

Furthermore, among the variables that can confound the conclusions in this type of investigation, is the method used to calculate the dependent variable, in this case, the risk score. In this way, based on similar analysis (Brouwer et al., 2013; Ekelund et al., 2007), we adopted an indicator both with and without waist circumference. The primary reason for analysing risk without the indicator of central adiposity is related to the fact that this is the most prevalent risk factor and the most closely related to body fat. In the same way, we observed that, independent of central adiposity, both exposures were related to the risk score; however, 

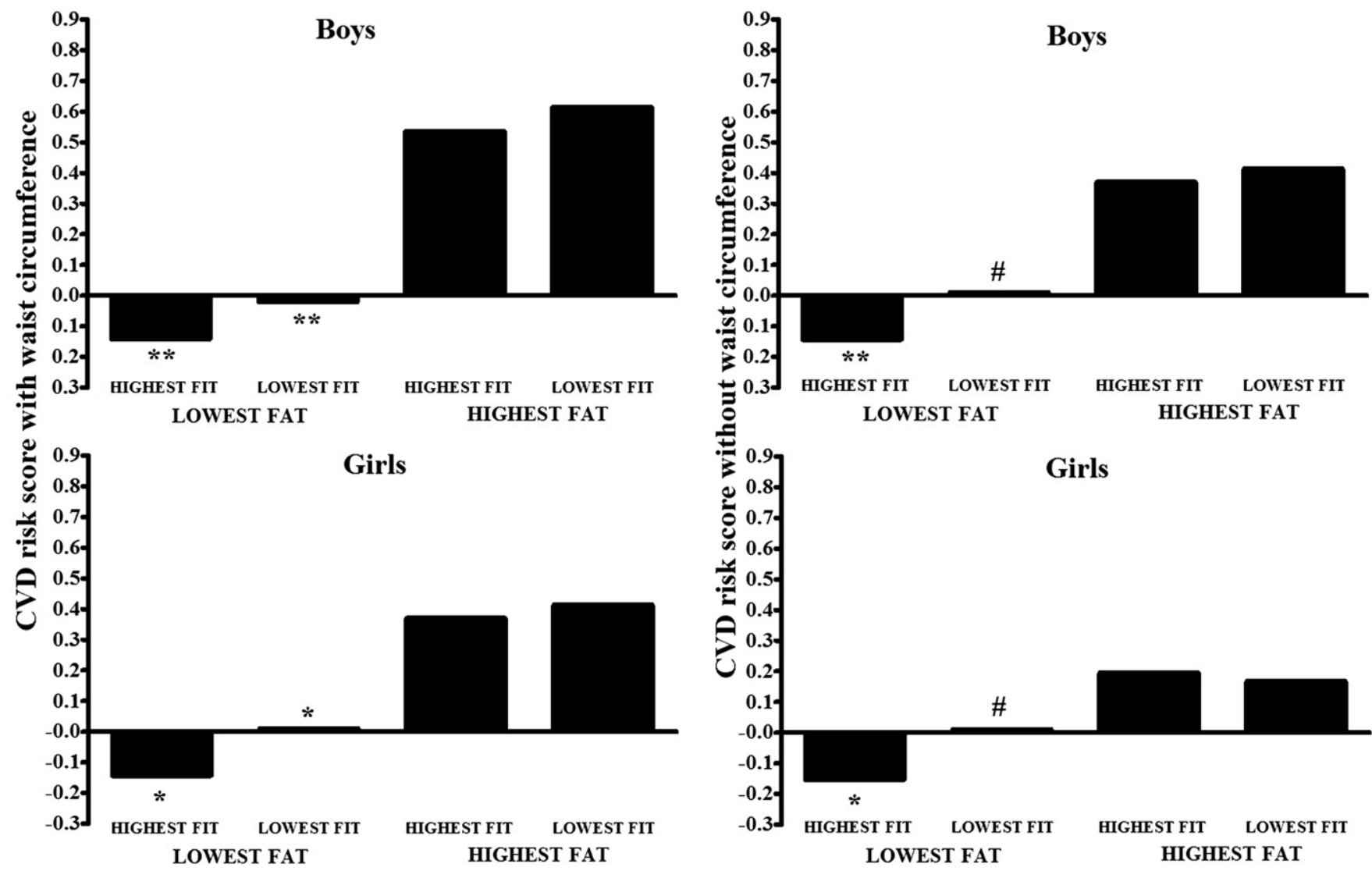

Figure 1. CVD risk score (with and without waist circumference) according to fat/fit categories in boys and girls. WC, waist circumference. *Difference between all groups. **Difference vs highest fit/highest fat and lowest fit/highest fat. \#Differences vs highest fat/lowest fit. Adjusted by chronological age and age of peak height velocity.

Table 2. Crude and multivariate regression models for CVD risk (with and without waist circumference) prediction.

\begin{tabular}{|c|c|c|c|c|c|c|c|c|c|c|c|c|}
\hline & \multicolumn{6}{|c|}{ CVD risk score with waist circumference } & \multicolumn{6}{|c|}{ CVD risk score without waist circumference } \\
\hline & \multicolumn{3}{|c|}{ Boys } & \multicolumn{3}{|c|}{ Girls } & \multicolumn{3}{|c|}{ Boys } & \multicolumn{3}{|c|}{ Girls } \\
\hline & $R_{\text {adj }}^{2}$ & $\beta$ & $p$ & $R_{\text {adj }}^{2}$ & $\beta$ & $p$ & $R_{\text {adj }}^{2}$ & $\beta$ & $p$ & $R_{\text {adj }}^{2}$ & $\beta$ & $p$ \\
\hline \multicolumn{13}{|l|}{ Crude analyses } \\
\hline Body fat & .343 & .050 & $<.001$ & .237 & .042 & $<.001$ & 173 & .035 & $<.001$ & .066 & .023 & $<.001$ \\
\hline $\mathrm{VO}_{2}$ peak & .155 & -.050 & $<.001$ & .053 & -.032 & $<.001$ & .104 & -.040 & $<.001$ & .018 & -.020 & .001 \\
\hline Adjusted analyses & .477 & & & .286 & & & .253 & & & .106 & & \\
\hline Body fat & & .038 & $<.001$ & & .031 & $<.001$ & & .023 & $<.001$ & & .012 & .004 \\
\hline $\mathrm{VO}_{2}$ peak & & -.013 & .013 & & -.016 & .017 & & -.017 & .007 & & -.019 & .011 \\
\hline
\end{tabular}

cardiorespiratory fitness seemed to have a stronger relationship with the final score, when compared with the score traditionally adopted in previous studies. This may explain, in part, some differences between the studies, especially the predominance of body fat relating to cardiorespiratory fitness. Eisenmann et al. (2007), for example, observed that cardiorespiratory fitness offered protection to cardiovascular risk, mainly in obese individuals. However, based on our findings, since the adopted outcome included waist circumference, it is probable that the strength of adiposity was over-estimated.

Multivariate models help us better understand the relationship between variables. First, the coefficients of determination were always greater for the indicator of risk with waist circumference, a fact explained by the more direct relationship between the indicators of adiposity (previously referred to). Second, the coefficients were always higher in boys, supporting previous findings (Cummings et al., 2010; Musa \& Williams, 2012) and indicating that, in girls, other variables may be more related to risk. Finally, the beta coefficient modifications, according to the outcome (with or without waist circumference), were different for body fat and cardiorespiratory fitness; in other words, while the coefficient of body fat decreased, the cardiorespiratory fitness coefficient increased with the removal of waist circumference from the risk, independent of sex. These results indicate the importance of cardiorespiratory fitness in the genesis of pathological conditions with a greater latency period, especially when we consider the stability of these indicators over time (Eisenmann et al., 2004). 
Interpretation of these data could assist the formulation of strategies for the promotion of health in young people. If, on one hand, the treatment of obesity requires a complex multifactorial approach and radical changes are related to the failure of interventions, we suggest that gradual changes in the level of physical activity and sedentary behaviour can affect health through the increase in cardiorespiratory fitness. In the present study, we observed that the adolescents with high body fat, who presented high cardiorespiratory fitness, presented protection for the cardiovascular risk indicators. On the other hand, the treatment of obesity through actions that do not improve cardiorespiratory fitness, especially drug interventions, should be rethought, since we observed that not only body fat explained the risk in this population. Besides cardiorespiratory fitness, Artero et al. (2014) also highlighted the role of muscular fitness in the maintenance of a good inflammatory profile, independent of obesity, emphasising the relationship between a global profile of physical fitness and cardiovascular risk. In this way, we suggest that the promotion of physical activity should be focused not only on prevention and treatment of obesity, but the wide range of components that mediate its benefits.

Regarding the methodological aspects adopted in this study, the 20 metre shuttle run test was used to estimate cardiorespiratory fitness (Cummings et al., 2010; Eisenmann et al., 2007; Musa \& Williams, 2012) and seems to have a good relationship with laboratory tests (Batista et al., 2013). With respect to metabolic risk, given the criticisms of the categorical concept of metabolic syndrome (Eisenmann, 2008), we opted for a continuous score, which also favours the assessment of gradual relationships. For estimating body fat, we adopted skinfold thickness, which is a widely used method in epidemiologic studies (Andersen et al., 2008; Ekelund et al., 2007), that, when controlling for the quality of measures, provides confident estimates. For biological maturation, we adopted the age of peak height velocity (Mirwald et al., 2002), an objective indicator accepted for evaluation of somatic maturation. Finally, given our design characteristic, no establishment of temporality between the variables was possible.

Thus, we conclude that both body fat and cardiorespiratory fitness are related to cardiovascular risk in adolescents; however, the strength of this relationship tends to vary according to the composition of the adopted risk indicator.

\section{Acknowledgements}

The authors thank Alessandra Okino, Jair Oliveira, and Danielle Venturini for research support and Crisieli Tomeleri, Mariana Carnelossi and Sandra Kawaguti for acquisition of data. This study was funded by the National Council of Scientific and Technological Development (CNPq/Brazil - Process 483867/2009-8). ERVR, ESC and RAF were supported by grants from CNPq/Brazil. DRS (sandwich doctorate scholarship - Process 201022/2015-0) and AOW (scientific initiation) are also supported by CNPq. Contract grant sponsor: Brazilian Council of Scientific and Technological Development (483867/2009-8). Informed consent was obtained from all individual participants included in the study.

\section{Disclosure statement}

The authors report no conflicts of interest. The authors alone are responsible for the content and writing of the paper.

\section{Funding}

Conselho Nacional de Desenvolvimento Científico e Tecnológico, 10.13039/501100003593 [483867/2009-8].

\section{References}

Andersen LB, Sardinha LB, Froberg K, Riddoch CJ, Page AS, Andersen SA. 2008 Fitness, fatness and clustering of cardiovascular risk factors in children from Denmark, Estonia and Portugal: The European Youth Heart Study. Int J Pediatr Obes 3:58-66.

Artero EG, España-Romero V, Jiménez-Pavón D, Martinez-Gómez D, Warnberg J, Gómez-Martínez S, et al. 2014. Muscular fitness, fatness and inflammatory biomarkers in adolescents. Pediatr Obes 9:391-400.

Barlow CE, Kohl HW, Gibbons LW, Blair SN. 1995. Physical fitness, mortality and obesity. Int J Obes Relat Metab Disord 19(Suppl 4): S41-S44.

Batista MB, Cyrino ES, Arruda M, Dourado AC, Coelho ESMJ, Ohara D, et al. 2013. Validity of equations for estimating VO2peak from the 20$\mathrm{m}$ shuttle run test in adolescents aged 11-13 years. J Strength Cond Res 27:2774-2781.

Boileau RA, Lohman TG, Slaughter MH. 1985. Exercise body composition in children and youth. Scand J Sport Sci 7:17-27.

Brazilian Institute of Geography and Statistics (BIGS). 2013. Gross domestic product of municipalities. Available online at: http://www.ibge.gov. $\mathrm{br} /$ cidadesat/xtras/perfil.php?codmun=411370\&search=parana|londrina, (accessed July 5, 2013).

Brouwer SI, Stolk RP, Liem ET, Lemmink KA, Corpeleijn E. 2013. The role of fitness in the association between fatness and cardiometabolic risk from childhood to adolescence. Pediatr Diabetes 14:57-65.

Christofaro DGD, Fernandes RA, Gerage AM, Alves MJ, Polito MD, Oliveira ARD. 2009. Validação do monitor de medida de pressão arterial Omron HEM 742 em adolescentes. Arq Bras Cardiol 92:10-15.

Cummings DM, Dubose KD, Imai S, Collier DN. 2010. Fitness versus fatness and insulin resistance in U.S. Adolescents. J Obes 2010. doi:10.1155/2010/195729.

DuBose KD, Eisenmann JC, Donnelly JE. 2007. Aerobic fitness attenuates the metabolic syndrome score in normal-weight, at-risk-for-overweight, and overweight children. Pediatrics 120:1262-1268.

Eisenmann JC. 2007. Aerobic fitness, fatness and the metabolic syndrome in children and adolescents. Acta Paediatr 96:1723-1729.

Eisenmann JC. 2008. On the use of a continuous metabolic syndrome score in pediatric research. Cardiovasc Diabetol 7:1-6.

Eisenmann JC, Katzmarzyk PT, Perusse L, Tremblay A, Després JP, Bouchard C. 2005. Aerobic fitness, body mass index, and CVD risk factors among adolescents: the Québec family study. Int J Obes (Lond) 29:1077-1083.

Eisenmann JC, Welk GJ, Ihmels M, Dollman J. 2007. Fatness, fitness, and cardiovascular disease risk factors in children and adolescents. Med Sci Sports Exerc 39:1251-1256.

Eisenmann JC, Welk GJ, Wickel EE, Blair SN. 2004. Stability of variables associated with the metabolic syndrome from adolescence to adulthood: the Aerobics Center Longitudinal Study. Am J Hum Biol 16:690-696.

Ekelund U, Anderssen SA, Froberg K, Sardinha LB, Andersen LB, Brage S, European Youth Heart Study, G. 2007 Independent associations of physical activity and cardiorespiratory fitness with metabolic risk factors in children: the European youth heart study. Diabetologia 50:1832-1840.

Farrell SW, Cortese GM, LaMonte MJ, Blair SN. 2007. Cardiorespiratory fitness, different measures of adiposity, and cancer mortality in men. Obesity 15:3140-3149. 
Harrison G, Buskirk E, Carter L, Johnston F, Lohman T, Pollock M. 1988. Skinfold thicknesses and measurement technique. In: Lohman $T$, Roche A, Martorell R, editors. Anthropometric standardization reference manual. Champaign, IL: Human Kinetics Books. p 55-70.

Katzmarzyk PT, Srinivasan SR, Chen W, Malina RM, Bouchard C, Berenson GS. 2004. Body mass index, waist circumference, and clustering of cardiovascular disease risk factors in a biracial sample of children and adolescents. Pediatrics 114:e198-e205.

Kuk JL, Lee S. 2010. Independent associations between cardiorespiratory fitness and abdominal obesity with metabolic risk in adolescents and adults. Obesity (Silver Spring) 18:2061-2063.

Kwon S, Burns TL, Janz K. 2010. Associations of cardiorespiratory fitness and fatness with cardiovascular risk factors among adolescents: the NHANES 1999-2002. J Phys Act Health 7:746-753.

Lee YS. 2009. Consequences of childhood obesity. Ann Acad Med Singapore 38:75-77.

Leger LA, Lambert J. 1982. A maximal multistage 20-m shuttle run test to predict vo2 max. Eur J Appl Physiol 49:1-12.

Leger LA, Mercier D, Gadoury C, Lambert J. 1988. The multistage 20 metre shuttle run test for aerobic fitness. J Sports Sci 6:93-101.

Machado-Rodrigues AM, Leite N, Coelho-e-Silva MJ, Martins RA, Valentedos-Santos J, Mascarenhas LPG, et al. 2014. Independent association of clustered metabolic risk factors with cardiorespiratory fitness in youth aged 11-17 years. Ann Hum Biol 41:271-276.

Malina RM, Bouchard C, Bar-Or O. 2004. Growth, maturation, and physical activity. 2nd ed. Human Kinetics.

Mirwald RL, Baxter-Jones AD, Bailey DA, Beunen GP. 2002. An assessment of maturity from anthropometric measurements. Med Sci Sports Exerc 34:689-694.

Musa DI, Williams CA. 2012. Cardiorespiratory fitness, fatness, and blood pressure associations in Nigerian youth. Med Sci Sports Exerc 44:1978-1985.

Silva G, Aires L, Martins C, Mota J, Oliveira J, Ribeiro JC. 2013. Cardiorespiratory fitness associates with metabolic risk independent of central adiposity. Int J Sports Med 34:912-916.

Stabelini Neto A, Sasaki JE, Mascarenhas LPG, Boguszewski MCS, Bozza R, Ulbrich AZ, et al. 2011. Physical activity, cardiorespiratory fitness, and metabolic syndrome in adolescents: a cross-sectional study. Bmc Public Health 11:1-7

Suriano K, Curran J, Byrne SM, Jones TW, Davis EA. 2010. Fatness, fitness, and increased cardiovascular risk in young children. J Pediatr 157:552-558.

Wisloff U, Najjar SM, Ellingsen O, Haram PM, Swoap S, Al-Share Q, et al. 2005. Cardiovascular risk factors emerge after artificial selection for low aerobic capacity. Science 307:418-420. 